\title{
Expression of LDH and CEA in serum in the process of targeted therapy of lung adenocarcinoma and the association between them and prognosis
}

\author{
GUANGSI HE, ZONGHUI JIANG, SONG XUE, XIAOYAN SUN and WEIFEI WANG
}

The Second Department of Oncology, The First People's Hospital of Chuzhou, Chuzhou, Anhui 239000, P.R. China

Received September 19, 2018; Accepted January 29, 2019

DOI: 10.3892/ol.2019.10115

\begin{abstract}
Expression of lactate dehydrogenase (LDH) and carcinoembryonic antigen (CEA) in serum was investigated in the process of epidermal growth factor receptor-tyrosine kinase inhibitor (EGFR-TKI) targeting for treating lung adenocarcinoma and the association between LDH, CEA and prognosis of patients was evaluated. A retrospective analysis of 89 patients with lung adenocarcinoma admitted to The First People's Hospital of Chuzhou from January 2014 to February 2015 was performed. Fifty-one patients who received resection were considered the operation group, while the other 38 patients received EGFR-TKI targeted therapy and were considered the targeted group. Electrochemiluminescence and automatic biochemical analyzer were respectively used to detect the expression of CEA and LDH in serum. The therapeutic effective rates and the expression levels of LDH and CEA of the patients were compared. The patients in the targeted group were divided into LDH high-expression group, LDH low-expression group, CEA high-expression group and CEA low-expression group according to the median of the expression levels of LDH and CEA. The therapeutic effective rate in LDH high-expression group $(65.00 \%)$ was significantly lower than that in LDH low-expression group $(100.00 \%)(\mathrm{P}=0.004)$. The therapeutic effective rate in CEA high-expression group $(64.71 \%)$ was significantly lower than that in CEA low-expression group $(95.24 \%)(\mathrm{P}=0.016)$. The 3 -year overall mortality rate in LDH high-expression group (47.37\%) was significantly higher than that in LDH low-expression group
\end{abstract}

Correspondence to: Dr Guangsi He, The Second Department of Oncology, The First People's Hospital of Chuzhou, 12 Zhongyou Lane, Chuzhou, Anhui 239000, P.R. China

E-mail: he83nv@163.com

Key words: lactate dehydrogenase, carcinoembryonic antigen, lung adenocarcinoma, EGFR-TKI targeted therapy, therapeutic effective rate, prognosis
(11.11\%) $(\mathrm{P}=0.034)$. The 3-year overall mortality rate in CEA high-expression group $(56.25 \%)$ was significantly higher than that in CEA low-expression group (4.76\%) $(\mathrm{P}=0.020)$. The levels of CEA and LDH in serum were abnormally expressed in the process of the treatment of lung adenocarcinoma targeted by EGFR-TKI, which had great significance for monitoring the efficacy and prognosis of the treatment of lung adenocarcinoma targeted by EGFR-TKI.

\section{Introduction}

Lung cancer is one of the most common cancers in clinic, it is often found in mucosal epithelium of bronchus (1). Studies showed that in 2016 new patients with lung cancer increased $\sim 2.67$ million worldwide, and the morbidity of lung cancer ranked the second among all cancers (2). Lung adenocarcinoma is a lung cancer where the age of onset tends to be younger (3). At present, the pathogenesis of lung adenocarcinoma is still unclear. Tumor metastasis easily occurs in lung adenocarcinoma and $\sim 30 \%$ of patients with advanced lung adenocarcinoma have metastasis, which makes the fatality rate of lung adenocarcinoma the highest among all cancers $(4,5)$. It has been shown that without effective treatment, the median survival time of this type of patients is only 1 month (6). The most effective means of treating lung adenocarcinoma in clinic are resection, radiotherapy and chemotherapy (7). At present, with the development of the treatment of tumor diseases, lung cancer resection has been improved and nidus separation can be finished preferably in patients during resection (8), however, in the process of postoperative chemotherapy, side effects easily appear when normal lung tissue in the radiation field is injured (9). Patients with slight injury have difficulty in breathing and impairment of lung function, while in severe cases patients present extensive pulmonary fibrosis, which directly threatens their life (10). Therefore, the way to improve the therapeutic effective rate of lung adenocarcinoma is a major focus in current clinical research. Molecular targeted therapy has become a hotspot in research. Some data have demonstrated that AFAP1-AS1, CDK4, CDK6 and NAT8L may become potential targets of future clinical treatment of lung cancer (11-13). Epidermal growth factor receptor-tyrosine kinase inhibitor (EGFR-TKI) 
has an extremely important influence on lung adenocarcinoma (14). Cancer factors enhance the susceptibility of tumor tissue for programmed cell death protein 1 (PD-1) blockade via EGFR pathway, and the key of EGFR-TKI is inhibiting the combination of PD-1 and EGFR (15). It has been proven that lung adenocarcinoma receives the largest beneficial effect when EGFR-TKI is applied in the treatment of non-small cell carcinoma $(16,17)$, but it is still controversial how to better predict the clinical efficacy and prognosis of patients. Carcinoembryonic antigen (CEA) is currently the most sensitive tumor marker (18), and lactate dehydrogenase (LDH) is a prognostic indicator that is closely related to tumor cell load and distant metastasis (19). There are few studies on the two in the targeted therapy of lung adenocarcinoma, therefore, this report retrospectively analyzed patients with lung adenocarcinoma who were admitted and treated in The First People's Hospital of Chuzhou (Chuzhou, China) since 2014 to investigate the significance of CEA and LDH in the targeted therapy of lung adenocarcinoma.

\section{Patients and methods}

General data. Eighty-nine patients with lung adenocarcinoma, who were admitted to The First People's Hospital of Chuzhou from January 2014 to February 2015, were the study subjects and were analyzed retrospectively. Among the patients, there were 38 patients with EGFR sensitive mutation, accounting for $42.70 \%$ of all subjects. There were 34 males and 55 females, aged from 37 to 69 years, with an average age of $51.63 \pm 11.74$ years.

Inclusion and exclusion criteria. Inclusion criteria: all patients who were diagnosed with lung adenocarcinoma via biopsy in the Pathology Department of The First People's Hospital of Chuzhou; patients who received lung tissue resection or EGFR-TKI targeted therapy in The First People's Hospital of Chuzhou; patients with complete clinical data; patients 40-65 years of age. Exclusion criteria: patients with other abnormalities in cardiopulmonary functions before the preoperative diagnosis; with other tumor diseases, blood diseases, immune diseases, or physical disabilities; who were chronically ill; patients in gestation period; patients who had surgical contraindications; patients who had received chemotherapy before operation; patients pathologically diagnosed with local or distant metastasis after operation. The study was approved by the Ethics Committee of The First People's Hospital of Chuzhou. Patients had complete clinical data and signed informed consents were obtained from the patients and/or the guardians.

Methods. Among the 89 study subjects, 51 patients received resection in The First People's Hospital of Chuzhou and were set as the operation group; the other 38 patients received EGFR-TKI targeted therapy in The First People's Hospital of Chuzhou, and were considered the targeted group. The operations were performed by the senior chief clinicians. The therapeutic drug was AZD9291, also known as Osimertinib, which is the third generation of EGFR-TKI. This compound is an irreversible mutant selective EGFR-TKI (exon 19 deletion type EGFRIC $_{50}=12.92 \mathrm{nM}, \mathrm{L} 858 \mathrm{R} / \mathrm{T} 790 \mathrm{M}$
EGFR IC $_{50}=11.44 \mathrm{nM}$, wild-type EGFR $\mathrm{IC}_{50}=493.8 \mathrm{nM}$ ), oral administration, $80 \mathrm{mg}$, PO QD.

Venous blood of patients $(5.0 \mathrm{ml})$ was taken before surgery, 4 weeks after the targeted therapy, or 1 week after surgery. CEA electrochemiluminescence kit (cat. no. LT13001; Quanzhou Lantu Biotechnology Co., Ltd., Quanzhou, China) was used to detect the expression of CEA in serum with UniCel DxI 800 immunoassay system (Beckman Coulter, Inc., Brea, CA, USA), and an automatic biochemical analyzer (Beckman Coulter, Inc., Shanghai, China) was used to detect the expression of LDH in serum.

Observation indicators. The clinical data of patients in the two groups included sex, age, weight, and course of disease. The therapeutic effective rate was calculated using the Response Evaluation Criteria in Solid Tumors (RECIST) of 2012 as a reference (20) and the patients were separated into four stages, based on complete response (CR), partial response (PR), stable disease (SD) and progressive disease (PD). All patients took imageological examination at the 5th week after the treatment, and the imageological results were used for the evaluation and reference of the final efficacy. Therapeutic effective rate $=$ (the number of patients who were graded in $\mathrm{CR}+\mathrm{PR}$ )/total number $\mathrm{x} 100 \%$. Patients in the targeted group were given a 3-year prognostic follow-up in the form of call, letter, hospital review and visit. The deadline of the follow-up was April 2018 and the ultimate event was the death of the patient. The 3-year survival rate of the patients was recorded.

Statistical analysis. SPSS 24.0 statistical software (Shanghai Yuchuang Network Technology Co., Ltd., Shanghai, China) was used to analyze and process all data; the enumeration data were expressed in the form of rate, and Chi-square $\left(\chi^{2}\right)$ test was used for their comparison between groups; the measurement data were expressed as the mean \pm standard deviation, and paired t-test was used for their comparison between groups. Kaplan-Meier survival analysis was used for the survival curves and log-rank test was used for the comparison of the survival rates. $\mathrm{P}<0.05$ was considered to indicate a statistically significant difference.

\section{Results}

Comparison of the clinical data. There were no significant differences concerning age, weight, course of disease, sex, smoking, drinking, place of residence, tumor metastasis and differentiated degree of patients between the two groups $(\mathrm{P}>0.050)$. This proves that there was comparability between the patients of the two groups. Details are shown in Table I.

Comparison of the therapeutic effective rate. In the targeted group, $52.63 \%$ of the cases were CR, $28.95 \%$ were PR, $13.16 \%$ were $\mathrm{SD}$, and $5.26 \%$ were $\mathrm{PD}$; the therapeutic effective rate was $81.58 \%$. In the operation group, $56.86 \%$ of the cases were CR, $25.49 \%$ were PR, $9.80 \%$ were SD, and $5.88 \%$ were $\mathrm{PD}$; the therapeutic effective rate was $82.35 \%$. The differences were found to be statistically not significant when the therapeutic effective rates of the two groups were compared (P>0.050) (Table II). 
Table I. Comparison of the patients' clinical data between the two groups [n (\%)].

\begin{tabular}{|c|c|c|c|c|}
\hline Characteristics & Targeted group $(\mathrm{n}=38)$ & Operation group $(\mathrm{n}=51)$ & $\chi^{2}$ or $\mathrm{t}$ & P-value \\
\hline Age (years) & $51.67 \pm 12.33$ & $50.84 \pm 11.96$ & 0.320 & 0.750 \\
\hline Weight $(\mathrm{kg})$ & $72.96 \pm 15.68$ & $74.24 \pm 16.07$ & 0.376 & 0.708 \\
\hline Course of disease (weeks) & $3.52 \pm 1.66$ & $3.70 \pm 1.54$ & 0.528 & 0.599 \\
\hline Sex & & & 0.045 & 0.831 \\
\hline Male & $15(39.47)$ & $19(37.25)$ & & \\
\hline Female & $23(60.53)$ & $32(62.75)$ & & \\
\hline Smoking & & & 0.163 & 0.686 \\
\hline Yes & $8(21.05)$ & $9(17.65)$ & & \\
\hline No & $30(78.95)$ & $42(82.35)$ & & \\
\hline Drinking & & & 0.112 & 0.738 \\
\hline Yes & $5(13.16)$ & $8(15.69)$ & & \\
\hline No & $33(86.84)$ & $43(84.31)$ & & \\
\hline Place of residence & & & 0.362 & 0.547 \\
\hline City & $31(81.58)$ & $44(86.27)$ & & \\
\hline Countryside & $7(18.42)$ & $7(13.73)$ & & \\
\hline Tumor metastasis & & & 0.056 & 0.813 \\
\hline Yes & $9(23.68)$ & $11(21.57)$ & & \\
\hline No & $29(76.32)$ & $40(78.43)$ & & \\
\hline Differentiated degree & & & 0.159 & 0.924 \\
\hline High & $13(34.21)$ & $16(31.37)$ & & \\
\hline Middle & $20(52.63)$ & $29(56.86)$ & & \\
\hline Low & $5(13.16)$ & $6(11.76)$ & & \\
\hline
\end{tabular}

Table II. Comparison of the therapeutic effective rate [n (\%)].

\begin{tabular}{lcccc}
\hline Items & Targeted group $(\mathrm{n}=38)$ & Operation group $(\mathrm{n}=51)$ & $\chi^{2}$ & P-value \\
\hline $\mathrm{CR}$ & $20(52.63)$ & $29(56.86)$ & 0.787 & 0.375 \\
$\mathrm{PR}$ & $11(28.95)$ & $13(25.49)$ & 0.132 & 0.716 \\
$\mathrm{SD}$ & $5(13.16)$ & $5(9.80)$ & 0.246 & 0.620 \\
PD & $2(5.26)$ & $4(7.84)$ & 0.016 & 0.900 \\
Effective rate $(\%)$ & 81.58 & 82.35 & 0.009 & 0.925 \\
\hline
\end{tabular}

$\mathrm{CR}$, complete response; $\mathrm{PR}$, partial response; $\mathrm{SD}$, stable disease; $\mathrm{PD}$, progressive disease.

Comparison of the expression levels of $L D H$ and CEA. The expression levels of LDH in the targeted group before and after treatment were $224.56 \pm 12.67$ and $163.75 \pm 9.24 \mathrm{U} / 1$, respectively; the expression levels of $\mathrm{LDH}$ in the operation group before and after treatment were $230.64 \pm 13.08$ and $160.54 \pm 9.87 \mathrm{U} / 1$, respectively. There was no significant difference in the LDH of the patients in the two groups before or after treatment $(\mathrm{P}>0.050)$. The expression level of LDH after treatment was lower than that before treatment $(\mathrm{P}<0.050)$ in both groups. The expression levels of CEA in the targeted group before and after treatment were $20.77 \pm 3.68$ and $3.54 \pm 0.62 \mathrm{ng} / \mathrm{ml}$, respectively; the expression levels of CEA in the operation group before and after treatment were $21.34 \pm 3.92$ and $3.27 \pm 0.70 \mathrm{ng} / \mathrm{ml}$, respectively.
There was no significant difference in CEA of patients in the two groups before or after treatment $(\mathrm{P}>0.050)$. The expression level of CEA after treatment was lower than that before treatment $(\mathrm{P}<0.050)$ in both groups (Figs. 1 and 2$)$.

Difference of efficacy of the targeted groups. According to the medians of LDH and CEA expression levels in patients of the two groups before treatment, 20 cases were in LDH high-expression group ( $\mathrm{LDH} \geq 224.56 \mathrm{U} / \mathrm{l})$; 18 patients were in LDH low-expression group $(\mathrm{LDH}<224.56 \mathrm{U} / 1) ; 17$ patients were in CEA high-expression group $(\mathrm{CEA} \geq 20.77 \mathrm{ng} / \mathrm{ml})$; and 21 patients were in CEA low-expression group $($ CEA $<20.77 \mathrm{ng} / \mathrm{ml})$. The therapeutic effective rate of LDH high-expression group was $65.00 \%$, which was significantly lower than that of 
Table III. Therapeutic effective rate of LDH high and low-expression groups [n (\%)].

\begin{tabular}{lccc}
\hline Items & LDH high-expression group $(\mathrm{n}=20)$ & LDH low-expression group $(\mathrm{n}=18)$ & $\chi^{2}$ \\
\hline CR & $5(25.00)$ & $14(77.78)$ \\
PR & $8(40.00)$ & $4(22.22)$ \\
SD & $5(25.00)$ & $0(0.00)$ \\
PD & $2(10.00)$ & $0(0.00)$ & 8.485 \\
Effective rate $(\%)$ & 65.00 & 100.00 & 0.004 \\
\hline
\end{tabular}

LDH, lactate dehydrogenase; CR, complete response; $\mathrm{PR}$, partial response; SD, stable disease; PD, progressive disease.

Table IV. Therapeutic effective rate of CEA high and low-expression groups [n (\%)].

\begin{tabular}{lccc}
\hline Items & CEA high-expression group $(\mathrm{n}=17)$ & CEA low-expression group $(\mathrm{n}=21)$ & $\chi^{2}$ \\
\hline CR & $5(29.41)$ & $15(71.43)$ \\
PR & $6(35.29)$ & $5(23.81)$ \\
SD & $4(23.53)$ & $1(4.76)$ \\
PD & $2(11.76)$ & $0(0.00)$ & 5.828 \\
Effective rate $(\%)$ & 64.71 & 95.24 & 0.016 \\
\hline
\end{tabular}

CEA, carcinoembryonic antigen; CR, complete response; PR, partial response; SD, stable disease; PD, progressive disease.

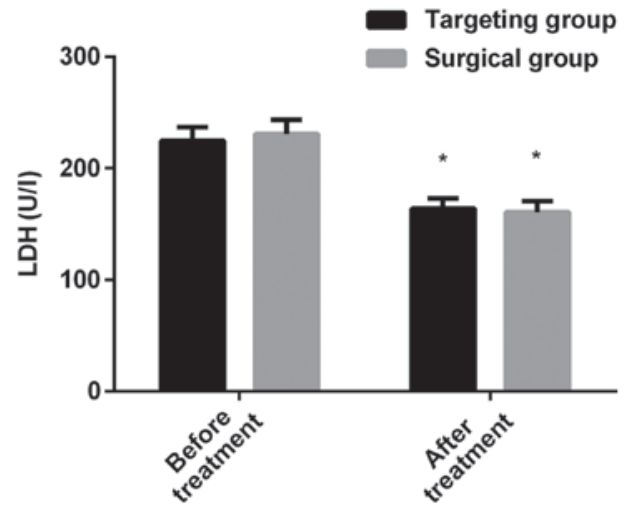

Figure 1. Comparison of the expression level of LDH between the targeted and the operation group before and after treatment. The level of LDH in the serum of the patients was detected by using an automatic biochemical analyzer. The results showed that there was no significant difference in the expression level of $\mathrm{LDH}$ between the two groups before or after treatment. The expression level of LDH after treatment decreased in both groups when compared to that before treatment. ${ }^{*} \mathrm{P}<0.050$, compared with the expression level of $\mathrm{LDH}$ in the same group before treatment. LDH, lactate dehydrogenase.

LDH low-expression group $(100.00 \%)(\mathrm{P}=0.004)$. The therapeutic effective rate of CEA high-expression group was $64.71 \%$, which was also significantly lower than that of CEA low-expression group (95.24\%) $(\mathrm{P}=0.016)$. Details are shown in Tables III and IV.

Prognosis. Among the 38 patients in the targeted group, 37 patients were successfully followed up, and the success rate of the follow-up visit was $97.37 \%$. There were 19 patients in LDH high-expression group, 18 patients in LDH low-expression

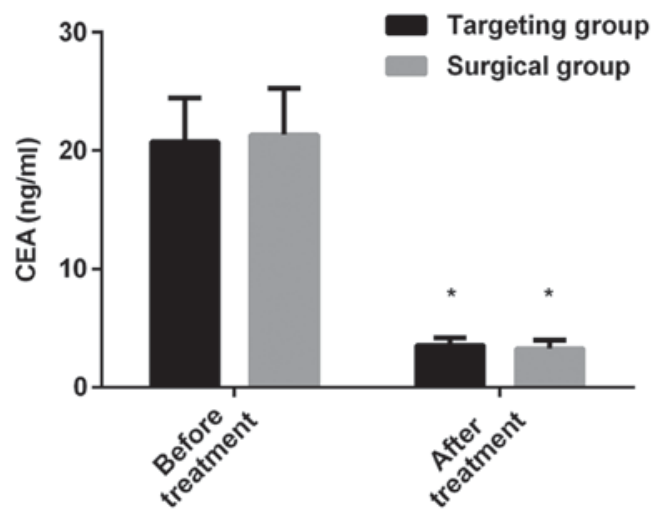

Figure 2. Comparison of the expression level of CEA between the targeted and the operation group before and after treatment. The level of CEA in the serum of the patients was detected by electrochemiluminescence. The results showed that there was no significant difference in the expression level of CEA between the two groups before or after treatment. The expression level of CEA after treatment decreased in both groups when compared to that before treatment. ${ }^{*} \mathrm{P}<0.050$, compared with the expression level of CEA in the same group before treatment. CEA, carcinoembryonic antigen.

group, 16 patients in CEA high-expression group and 21 patients in CEA low-expression group. The 3-year total mortality of LDH high-expression group was $47.37 \%$, which was significantly higher than that of LDH low-expression group (11.11\%) $(\mathrm{P}=0.034)$. The 3-year total mortality of CEA high-expression group was $56.25 \%$, which was significantly higher than that of CEA low-expression group (4.76\%) $(\mathrm{P}=0.020)$. When the data of all the deceased patients were compared, it was concluded that 14 patients $(77.78 \%)$ had high-expression of LDH and CEA (Figs. 3 and 4). 


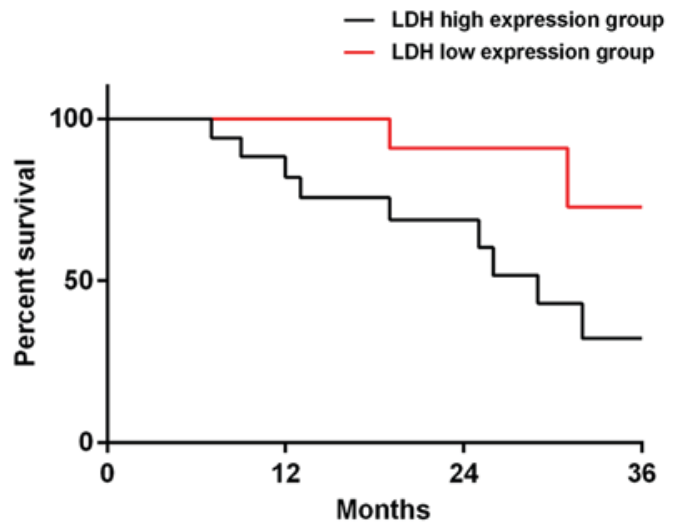

Figure 3. The survival curves of LDH high and low-expression groups at 3 years after prognosis. The total mortality of LDH high-expression group at 3 years was $47.37 \%$, which was significantly lower than that of LDH low-expression group $(11.11 \%, \mathrm{P}=0.034)$. $\mathrm{LDH}$, lactate dehydrogenase.

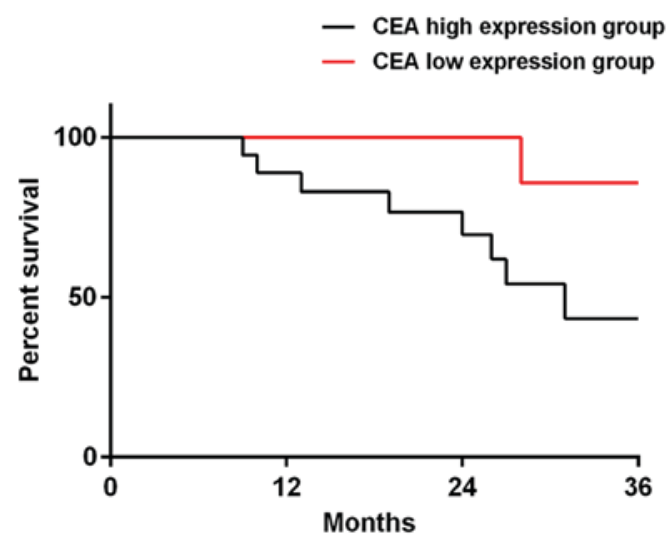

Figure 4. The survival curves of CEA high and low-expression groups at 3 years after prognosis. The total mortality of CEA high-expression group at 3 years was $56.25 \%$, which was significantly lower than that of CEA low-expression group $(4.76 \%, \mathrm{P}=0.020)$. CEA, carcinoembryonic antigen.

\section{Discussion}

The morbidity and mortality of lung adenocarcinoma are extremely high in clinic (21). The scheme of diagnosis and treatment of lung adenocarcinoma has long been a research hotspot in clinic, and targeted therapy has gradually become popularized in recent years. Compared with traditional resection, radiotherapy and chemotherapy, the targeted therapy is safer in ensuring the effective remission of patients' tumors, and it achieves the purpose of treating tumors by regulating one or more factors directly related to tumors $(21,22)$. While EGFR-TKI is applied in lung adenocarcinoma, the mechanism is using the combination between gefitinib or erlotinib and EGFR in lung adenocarcinoma cells, and disrupting the signal transduction of the downstream cells by inhibiting the activation of tyrosine kinase, thereby preventing the spread, infiltration and metastasis of cancer cells (23). However, some related drug resistance usually appears in most patients in the process of targeted therapy, which weakens the therapeutic effect of targeted drugs (24). So it is particularly important to monitor the therapeutic effect and estimate the condition of prognosis in the process of targeted therapy.
In this study, the difference of the efficacy between EGFR-TKI targeted therapy and traditional resection for the patients with lung adenocarcinoma, and the expression levels of LDH and CEA were investigated. The significance of the expression levels of LDH and CEA and the effect of prognosis of LDH and CEA in patients with lung adenocarcinoma who were treated with EGFR-TKI targeted therapy were also investigated. Results showed that there is no significant difference in the therapeutic effective rates and the expression of LDH and CEA between the targeted and the operation group, which suggests that EGFR-TKI targeted therapy has an extremely high application value for treating lung adenocarcinoma. LDH mainly belongs to the enzyme in the glycolytic pathway and plays a great role in facilitating the formation of lactic acid, which originates from pyruvate (25). In general, LDH exists in almost every cell of the organisms, and its activity is generally high in human body. Therefore, LDH does not have high specificity for the early diagnosis of diseases, and it is often used as a reference to prognostically monitor and estimate the state of the disease of patients (26). CEA is a glycoprotein that only exists in cancer and embryonic tissues, and directly acts on the adhesion reaction between tumor cells, which has a strong regulatory ability on the differentiation, apoptosis, infiltration and metastasis of cancer cells (27). In this study, there was no difference between the targeted and the operation group, which proves that EGFR-TKI targeted therapy could effectively improve the condition of tumor nidus of lung adenocarcinoma in patients, reduce the tumor volume in patients, and achieve the purpose of treating tumors. In addition, there was no difference in the expression of CEA and LDH before or after treatment between the two groups, which preliminarily suggests that these indicators could be generally applied for the treatment of lung cancer, and errors in expression levels would not be caused by traumatic surgery. However, more in-depth research is required for comparison in order to obtain most accurate experiment results. The contingency could not be excluded due to the small sample size in this study. Analysis of the efficacy and the difference of prognosis of patients in CEA high-expression group, CEA low-expression group, LDH high-expression group and LDH low-expression group in the targeted group showed that CEA and LDH high-expression groups were both significantly worse than CEA and LDH low-expression groups. This suggests that CEA and LDH both have a high application value for monitoring the efficacy and prognosis of patients who receive EGFR-TKI targeted therapy. Tumor cells can perform unlimited aerobic glycolysis with the support of enough nutrition, while the main substance that provides nutrition for them is LDH (28). It is speculated that due to the incomplete elimination of tumor nidus, cancer cells consume a large amount of oxygen in patient's body, thus the patient's body is chronically in a state of hypoxia, which induces the enhancement of glycolysis and raises the level of LDH, allowing tumor cells to get enough energy again to further metastasize and invade. So, the prognosis of LDH high-expression group is significantly worse than that of LDH low-expression group. CEA, a tumor marker, reflects the active ability of tumors. The higher its expression is, the stronger the activation ability of tumors is, thereby, the prognosis is worse.

In this study, the difference of efficacy between EGFR-TKI targeted therapy and traditional resection in the treatment of patients with lung adenocarcinoma and the expression of LDH 
and CEA were investigated, and the significance of the expression and the effect of prognosis of LDH and CEA in patients with lung adenocarcinoma treated with EGFR-TKI targeted therapy were also investigated. There were some limitations because of the lack of experimental conditions. For example, the sample size was small, and the experimental population was uniform. Also, the time of the follow-up was short. LDH and CEA are both reactants with low specificity, and the abnormal expression of them could occur in patients with severe infections and organ damage.

In conclusion, the levels of CEA and LDH in serum are abnormally expressed in the process of EGFR-TKI targeted therapy of lung adenocarcinoma, which is of great significance for monitoring the efficacy and prognosis of the therapy of lung adenocarcinoma targeted by EGFR-TKI.

\section{Acknowledgements}

Not applicable.

\section{Funding}

No funding was received.

\section{Availability of data and materials}

The datasets used and/or analyzed during the present study are available from the corresponding author on reasonable request.

\section{Authors' contributions}

GH, ZJ and SX recorded and analyzed the observation indicators. XS and WW collected and interpreted the patients' data using electrochemiluminescence, the automatic biochemical analyzer and SPSS 24.0 statistical software. GH wrote and revised the manuscript. All authors read and approved the final manuscript.

\section{Ethics approval and consent to participate}

The study was approved by the Ethics Committee of The First People's Hospital of Chuzhou (Chuzhou, China). Patients who participated in this research had complete clinical data and signed informed consents were obtained from the patients and/or the guardians.

\section{Patient consent for publication}

Not applicable.

\section{Competing interests}

The authors declare that they have no competing interests.

\section{References}

1. Reck M, Rodríguez-Abreu D, Robinson AG, Hui R, Csőszi T, Fülöp A, Gottfried M, Peled N, Tafreshi A, Cuffe S, et al; KEYNOTE-024 Investigators: Pembrolizumab versus chemotherapy for PD-L1 - positive non-small-cell lung cancer. N Engl J Med 375: 1823-1833, 2016.
2. Herbst RS, Baas P, Kim DW, Felip E, Pérez-Gracia JL, Han JY, Molina J, Kim JH, Arvis CD, Ahn MJ, et al: Pembrolizumab versus docetaxel for previously treated,PD-L1-positive, advanced non-small-cell lung cancer (KEYNOTE-010): A randomised controlled trial. Lancet 387: 1540-1550, 2016.

3. Lou Y, Diao L, Cuentas ERP, Denning WL, Chen L, Fan YH, Byers LA, Wang J, Papadimitrakopoulou VA, Behrens C, et al: Epithelial-mesenchymal transition is associated with a distinct tumor microenvironment including elevation of inflammatory signals and multiple immune checkpoints in lung adenocarcinoma. Clin Cancer Res 22: 3630-3642, 2016.

4. Takada K, Okamoto T, Shoji F, Shimokawa M, Akamine T, Takamori S, Katsura M, Suzuki Y, Fujishita T, Toyokawa G, et al: Clinical significance of PD-L1 protein expression in surgically resected primary lung adenocarcinoma. J Thorac Oncol 11: 1879-1890, 2016

5. Johung KL, Yeh N, Desai NB, Williams TM, Lautenschlaeger T, Arvold ND, Ning MS, Attia A, Lovly CM, Goldberg S, et al: Extended survival and prognostic factors for patients with ALK-rearranged non-small-cell lung cancer and brain metastasis. J Clin Oncol 34: 123-129, 2016.

6. Schuler M, Wu YL, Hirsh V, O'Byrne K, Yamamoto N, Mok T, Popat S, Sequist LV, Massey D, Zazulina V, et al: First-line afatinib versus chemotherapy in patients with non-small cell lung cancer and common epidermal growth factor receptor gene mutations and brain metastases. J Thorac Oncol 11: 380-390, 2016.

7. Ambrogio C, Gómez-López G, Falcone M, Vidal A, Nadal E, Crosetto N, Blasco RB, Fernández-Marcos PJ, SánchezCéspedes M, Ren X, et al: Combined inhibition of DDR1 and Notch signaling is a therapeutic strategy for KRAS-driven lung adenocarcinoma. Nat Med 22: 270-277, 2016.

8. Martin JT, Durbin EB, Chen L, Gal T, Mahan A, Ferraris V and Zwischenberger J: Nodal upstaging during lung cancer resection is associated with surgical approach. Ann Thorac Surg 101: 238-244, discussion 44-45, 2016.

9. Kwon Y: Mechanism-based management for mucositis: Option for treating side effects without compromising the efficacy of cancer therapy. OncoTargets Ther 9: 2007-2016, 2016.

10. Chen QY, Jiao DM, Wang J, Hu H, Tang X, Chen J, Mou H and Lu W: miR-206 regulates cisplatin resistance and EMT in human lung adenocarcinoma cells partly by targeting MET. Oncotarget 7: 24510-24526, 2016.

11. Zeng Z, Bo H, Gong Z, Lian Y, Li X, Li X, Zhang W, Deng H, Zhou M, Peng S, et al: AFAP1-AS1, a long noncoding RNA upregulated in lung cancer and promotes invasion and metastasis. Tumour Biol 37: 729-737, 2016.

12. Patnaik A, Rosen LS, Tolaney SM, Tolcher AW, Goldman JW, Gandhi L, Papadopoulos KP, Beeram M, Rasco DW, Hilton JF, et al: Efficacy and safety of abemaciclib, an inhibitor of CDK4 and CDK6, for patients with breast cancer, non-small cell lung cancer, and other solid tumors. Cancer Discov 6: 740-753, 2016.

13. Lou TF, Sethuraman D, Dospoy P, Srivastva P, Kim HS, Kim J, Ma X, Chen PH, Huffman KE, Frink RE, et al: Cancer-specific production of $N$-acetylaspartate via NAT8L overexpression in non-small cell lung cancer and its potential as a circulating biomarker. Cancer Prev Res (Phila) 9: 43-52, 2016.

14. Wu SG, Liu YN, Tsai MF, Chang YL, Yu CJ, Yang PC, Yang JC, Wen YF and Shih JY: The mechanism of acquired resistance to irreversible EGFR tyrosine kinase inhibitor-afatinib in lung adenocarcinoma patients. Oncotarget 7: 12404-12413, 2016.

15. Ahn MJ, Sun JM, Lee SH, Ahn JS and Park K: EGFR TKI combination with immunotherapy in non-small cell lung cancer. Expert Opin Drug Saf 16: 465-469, 2017.

16. Zheng D, Ye X, Zhang MZ, Sun Y, Wang JY, Ni J, Zhang HP, Zhang L, Luo J, Zhang J, et al: Plasma EGFR T790M ctDNA status is associated with clinical outcome in advanced NSCLC patients with acquired EGFR-TKI resistance. Sci Rep 6: 20913, 2016.

17. Gainor JF, Shaw AT, Sequist LV, Fu X, Azzoli CG, Piotrowska Z, Huynh TG, Zhao L, Fulton L, Schultz KR, et al: EGFR mutations and ALK rearrangements are associated with low response rates to PD-1 pathway blockade in non-small cell lung cancer (NSCLC): A retrospective analysis. Clin Cancer Res 22: 4585-4593, 2016.

18. Bacac M, Fauti T, Sam J, Colombetti S, Weinzierl T, Ouaret D, Bodmer W, Lehmann S, Hofer T, Hosse RJ, et al: A novel carcinoembryonic antigen T-cell bispecific antibody (CEA TCB) for the treatment of solid tumor. Clin Cancer Res 22: 3286-3297, 2016.

19. Valvona CJ, Fillmore HL, Nunn PB and Pilkington GJ: The regulation and function of lactate dehydrogenase $\mathrm{A}$ : Therapeutic potential in brain tumor. Brain Pathol 26: 3-17, 2016. 
20. Shiono $S$ and Yanagawa N: Spread through air spaces is a predictive factor of recurrence and a prognostic factor in stage I lung adenocarcinoma. Interact Cardiovasc Thorac Surg 23: 567-572, 2016.

21. Minguet J, Smith KH and Bramlage P: Targeted therapies for treatment of non-small cell lung cancer - recent advances and future perspectives. Int J Cancer 138: 2549-2561, 2016.

22. Cha YJ, Kim HR, Lee CY, Cho BC and Shim HS: Clinicopathological and prognostic significance of programmed cell death ligand-1 expression in lung adenocarcinoma and its relationship with p53 status. Lung Cancer 97: 73-80, 2016.

23. Park K, Tan EH, O'Byrne K, Zhang L, Boyer M, Mok T, Hirsh V, Yang JC8 Lee KH, Lu S, et al: Afatinib versus gefitinib as first-line treatment of patients with EGFR mutation-positive non-small-cell lung cancer (LUX-Lung 7): A phase 2B, open-label, randomised controlled trial. Lancet Oncol 17: 577-589, 2016.

24. Yang JCH, Sequist LV, Zhou C, Schuler M, Geater SL, Mok T, Hu CP, Yamamoto N, Feng J, O'Byrne K, et al: Effect of dose adjustment on the safety and efficacy of afatinib for EGFR mutation-positive lung adenocarcinoma: Post hoc analyses of the randomized LUX-Lung 3 and 6 trials. Ann Oncol 27: 2103-2110, 2016.

25. Zhou M, Snedecor BR, Ng CKD and Shen A: Decreasing lactate level and increasing polypeptide production by downregulating the expression of lactate dehydrogenase and pyruvate dehydrogenase kinase: US Patent 9,487,809. Filed November 28, 2012; issued November 8, 2016.
26. Mohammad GH, Olde Damink SW, Malago M, Dhar DK and Pereira SP: Pyruvate kinase M2 and lactate dehydrogenase A are overexpressed in pancreatic cancer and correlate with poor outcome. PLoS One 11: e0151635, 2016.

27. Sørensen CG, Karlsson WK, Pommergaard HC, Burcharth J and Rosenberg J: The diagnostic accuracy of carcinoembryonic antigen to detect colorectal cancer recurrence - A systematic review. Int J Surg 25: 134-144, 2016

28. Koukourakis MI, Kakouratos C, Kalamida D, Bampali Z, Mavropoulou S, Sivridis E and Giatromanolaki A: Hypoxia-inducible proteins HIF1 $\alpha$ and lactate dehydrogenase LDH5, key markers of anaerobic metabolism, relate with stem cell markers and poor post-radiotherapy outcome in bladder cancer. Int J Radiat Biol 92: 353-363, 2016.

This work is licensed under a Creative Commons Attribution-NonCommercial-NoDerivatives 4.0 International (CC BY-NC-ND 4.0) License. 